\title{
Polarization Coupling Between Strongly Guiding Waveguides Stacked Laterally
}

\author{
Junji Yamauchi, Senior Member, IEEE, Member, OSA, Noriyuki Shibuya, and Hisamatsu Nakano, Fellow, IEEE
}

\begin{abstract}
The full-vectorial beam-propagation method is applied to the assessment of the coupling characteristics between strongly guiding waveguides stacked laterally. The polarization crosstalk behavior of square waveguides stacked laterally is demonstrated by the eigenmode and beam-propagation analyses. In order to make use of the polarization crosstalk constructively, we determine the vertical spacing between the two square waveguides. Almost complete conversion can be obtained, when the two waveguides are stacked diagonally. Another coupler composed of two rectangular waveguides is studied to realize the polarization conversion without exchanging the power with the same polarization. A crossing-waveguide-type polarization converter is also developed, with a short device length of less than $80 \mu \mathrm{m}$ at a wavelength of $1.55 \mu \mathrm{m}$.
\end{abstract}

Index Terms-Beam-propagation method, laterally stacked waveguides, polarization converter, polarization crosstalk.

\section{INTRODUCTION}

A DIRECTIONAL coupler plays significant roles in power division, modulation, switching, frequency selection, polarization selection, etc. The directional coupler is divided into two types, i.e., planar type and stacked type.

Vertical coupling between strongly guiding waveguides attracts attention for realizing optical compact circuits [1]-[3]. A simple eight-channel add-drop filter incorporating microring resonators vertically coupled to buried waveguides on a crossgrid array has been demonstrated [1], [2]. In [3], a simple optical add/drop multiplexers based on X-crossing vertical coupler filters with laterally separated input and output waveguides has been demonstrated. For a simple directional coupler, we are often faced with "polarization crosstalk", in which the polarization state changes as the field propagates. Little and Chu [2] have found that the polarization conversion occurs in a vertically coupled microring resonator. Furthermore, Fietz and Shvets have demonstrated that an on-chip dielectric microring resonator can be used to convert the polarization of light signals [4].

The purpose of this paper is to systematically reveal the polarization conversion behavior of strongly guiding waveguides

Manuscript received September 12, 2008; revised November 27, 2008. First published April 17, 2009; current version published July 01, 2009. This work was supported by MEXT Grant-in-Aid for Scientific Research (C) under Grant 19560355.

J. Yamauchi and H. Nakano are with the Faculty of Engineering, Hosei University, Koganei, Tokyo 184-8584, Japan (e-mail: j.yma@k.hosei.ac.jp).

N. Shibuya is with Sharp Corporation, Kameyama, Mie 519-0198, Japan. (e-mail: shibuya.noriyuki@sharp.co.jp).

Color versions of one or more of the figures in this paper are available online at http://ieeexplore.ieee.org.

Digital Object Identifier 10.1109/JLT.2008.2010782 stacked laterally. The full-vectorial beam-propagation method (FV-BPM) [5], [6] is used to numerically show the coupling characteristics in detail.

The eigenmodes of the coupler composed of two square cores are analyzed using the imaginary-distance FV-BPM. The polarization conversion length of the even (odd) supermodes is evaluated. The propagating beam analysis shows that the polarization conversion occurs due to the interference between the two even (odd) supermodes.

In order to make use of the polarization crosstalk constructively, we next determine the vertical spacing between the two square cores using the imaginary-distance FV-BPM. Almost complete conversion can be obtained, when the two cores are stacked diagonally. For the propagating beam analysis, the input transverse-magnetic (TM) mode field is converted into the transverse-electric (TE) mode field with a guided-mode power of $98 \%$.

Next, we analyze the coupler composed of two rectangular cores [7] so that the polarization of the input field may be converted without exchanging the power with the same polarization. For the eigenmode and propagating beam analyses, the polarization of the input field is converted, as the field propagates, while no significant interference occurs between the even and odd supermodes.

Finally, we study a crossing-waveguide-type polarization converter. A device length of less than $80 \mu \mathrm{m}$ is obtained, when the crossing angle is chosen to be around $2.2^{\circ}$ at a wavelength of $1.55 \mu \mathrm{m}$.

\section{NUMERICAL METHOD}

The FV-BPM is formulated using the coupled paraxial wave equations with the two transverse electric field components $E_{x}$ and $E_{y}[5]$

$$
\begin{aligned}
2 j k n_{0} \frac{\partial E_{x}}{\partial z}= & \frac{\partial}{\partial x}\left(\frac{1}{n^{2}} \frac{\partial}{\partial x}\left(n^{2} E_{x}\right)\right)+\frac{\partial^{2} E_{x}}{\partial y^{2}}+k^{2}\left(n^{2}-n_{0}^{2}\right) E_{x} \\
& +\frac{\partial}{\partial x}\left(\frac{1}{n^{2}} \frac{\partial}{\partial y}\left(n^{2} E_{y}\right)\right)-\frac{\partial^{2} E_{y}}{\partial x \partial y} \\
2 j k n_{0} \frac{\partial E_{y}}{\partial z}= & \frac{\partial}{\partial y}\left(\frac{1}{n^{2}} \frac{\partial}{\partial y}\left(n^{2} E_{y}\right)\right)+\frac{\partial^{2} E_{y}}{\partial x^{2}}+k^{2}\left(n^{2}-n_{0}^{2}\right) E_{y} \\
& +\frac{\partial}{\partial y}\left(\frac{1}{n^{2}} \frac{\partial}{\partial x}\left(n^{2} E_{x}\right)\right)-\frac{\partial^{2} E_{x}}{\partial y \partial x}
\end{aligned}
$$

where $k$ is the wavenumber in free space, $n$ is the refractive index profile, and $n_{0}$ is the reference refractive index to be appropriately chosen. In deriving (1) and (2), we assume that the refractive index varies slowly along the $z$-direction together with the slowly varying envelope approximation: $2 k n_{0}|\partial E / \partial z| \gg\left|\partial^{2} E / \partial z^{2}\right|$. As will be discussed later, we 


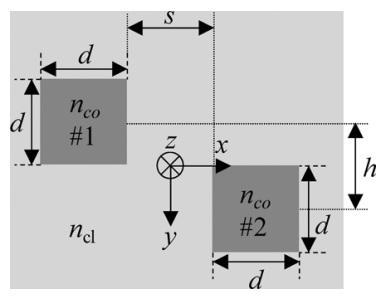

Fig. 1. Configuration of a coupler composed of two square cores.

treat a waveguide whose maximum tilt angle from the $z$-axis is $1.25^{\circ}$ in this paper. If a larger tilt angle is treated, a wide-angle formula will be desirable.

To discretize (1) and (2), we employ the pure Crank-Nicolson scheme [6], which has better stability than the McKee-Mitchell scheme [8]. The field discontinuities across the interfaces between different materials are taken into account using the improved finite-difference formulas [5].

The imaginary-distance BPM [9] is employed to analyze the eigenmodes of the coupler composed of two cores. In the imaginary-distance procedure, the propagation axis is taken to be the imaginary axis, i.e., $z=j \tau$. The propagation constant $\beta$ can easily be calculated by

$$
\beta=k n_{0}+\frac{\iint\left[\ln \left(E^{l+1}\right)-\ln \left(E^{l}\right)\right]\left|E^{l}\right|^{2} d x d y}{\Delta \tau \iint\left|E^{l}\right|^{2} d x d y}
$$

where $\Delta \tau$ and $l$ indicate the propagation step length and the position along the propagation direction, respectively.

Although exact TE and TM modes do not exist in 3-D waveguides, in this paper we simply use the expression of TE and TM modes, which corresponds to $E^{x}$ and $E^{y}$ modes, respectively.

\section{Coupler Composed of Two Square Cores}

Fig. 1 shows the cross section of the coupler whose two square cores are stacked laterally. The refractive indexes of the core and cladding are chosen to be $n_{\mathrm{co}}=2.2$ and $n_{\mathrm{cl}}=1.445$, respectively [10] (the relative refractive-index difference is calculated to be $\left.\Delta=\left(n_{\text {co }}^{2}-n_{\text {cl }}^{2}\right) / 2 n_{\text {co }}^{2} \simeq 28 \%\right)$. The width of the square core is taken to be $d=0.4 \mu \mathrm{m}$ for single-mode operation. The lateral spacing between the two cores is set to be $s=d=0.4 \mu \mathrm{m}$. The vertical displacement between the centers of the two cores is designated as $h$. Throughout this paper, the wavelength is fixed to be $\lambda=1.55 \mu \mathrm{m}$. The sampling widths arefixed to be $\Delta x=\Delta y=\Delta z=0.02 \mu \mathrm{m}$. In the imaginary-distance FV-BPM, the propagation step length is set to be $\Delta \tau=0.05 \mu \mathrm{m}$.

\section{A. Coupling Characteristics}

We first evaluate the coupling characteristics for $h=0.4 \mu \mathrm{m}$. In this case, the top side of Waveguide \#1 and the bottom side of Waveguide \#2 are in contact with the $x-z$ plane.

The eigenmode analysis shows the existence of two even supermodes $\left(\mathrm{S}_{\mathrm{e} 1}\right.$ and $\left.\mathrm{S}_{\mathrm{e} 2}\right)$ and two odd supermodes $\left(\mathrm{S}_{\mathrm{o} 1}\right.$ and $\mathrm{S}_{\mathrm{o} 2}$ ), as shown in Figs. 2 and 3, respectively. Note that the two even (odd) supermodes degenerate as the refractive index difference is decreased. Hence, for weakly guiding waveguides, power transfer occurs between the waveguides according to the
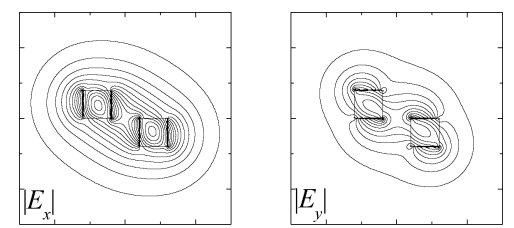

(a)
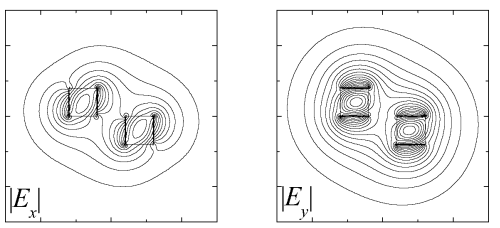

(b)

Fig. 2. Field distributions of the even supermodes. (a) $\mathrm{S}_{\mathrm{e} 1}$ mode. (b) $\mathrm{S}_{\mathrm{e} 2}$ mode.
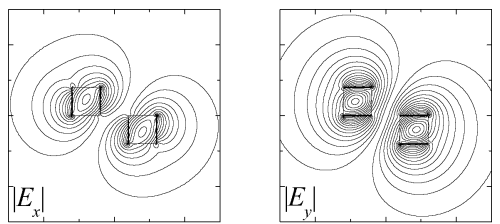

(a)
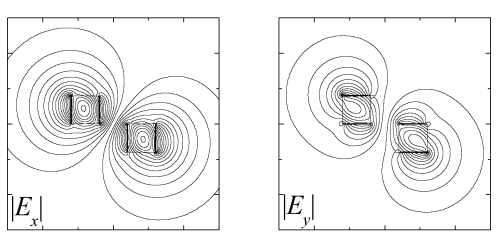

(b)

Fig. 3. Field distributions of the odd supermodes. (a) $\mathrm{S}_{\mathrm{o} 1}$ mode. (b) $\mathrm{S}_{\mathrm{o} 2}$ mode.

difference of the phase constants of the even and odd supermodes without generating polarization conversion. However, for strongly guiding waveguides, the phase constants of the two even (odd) supermodes are appreciably different, so that the interference between the two even (odd) supermodes occurs with polarization conversion. When one of the waveguides (e.g., Waveguide \#1) is excited with the fundamental TM mode of the isolated waveguide, the four supermodes are simultaneously excited. This leads to the power transfer between the two waveguides with polarization conversion, as the field propagates.

Next, we evaluate the polarization conversion lengths. The polarization conversion lengths obtained from the even and odd supermodes are, respectively, calculated as follows:

$$
\begin{aligned}
L_{\mathrm{p}(\text { even })} & =\frac{\pi}{\beta_{\mathrm{e} 1}-\beta_{\mathrm{e} 2}} \\
L_{\mathrm{p}(\text { odd })} & =\frac{\pi}{\beta_{\mathrm{o} 1}-\beta_{\mathrm{o} 2}}
\end{aligned}
$$

where $\beta_{\mathrm{e} 1}$ and $\beta_{\mathrm{e} 2}$ are the phase constants of the $\mathrm{S}_{\mathrm{e} 1}$ and $\mathrm{S}_{\mathrm{e} 2}$ supermodes, and $\beta_{\mathrm{o} 1}$ and $\beta_{\mathrm{o} 2}$ are those of the $\mathrm{S}_{\mathrm{o} 1}$ and $\mathrm{S}_{\mathrm{o} 2}$ supermodes, respectively. The eigenmode analysis shows that $L_{\mathrm{p}(\text { even })}=157 \mu \mathrm{m}$ and $L_{\mathrm{p}(\text { odd })}=303 \mu \mathrm{m}$.

We now evaluate the coupling characteristics, using the propagating beam analysis. Fig. 4 shows the normalized power of each field component as a function of propagation distance. 


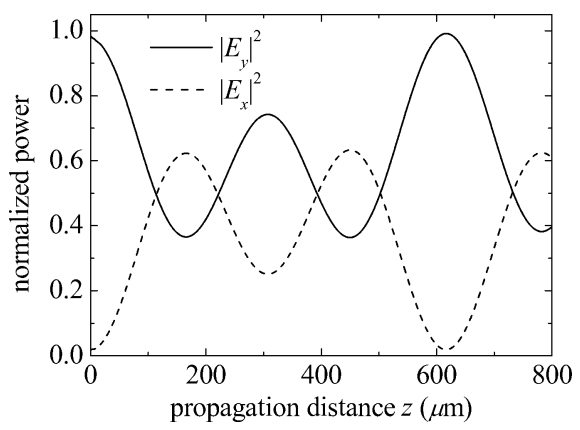

Fig. 4. Normalized power against propagation distance for $h=0.4 \mu \mathrm{m}$.

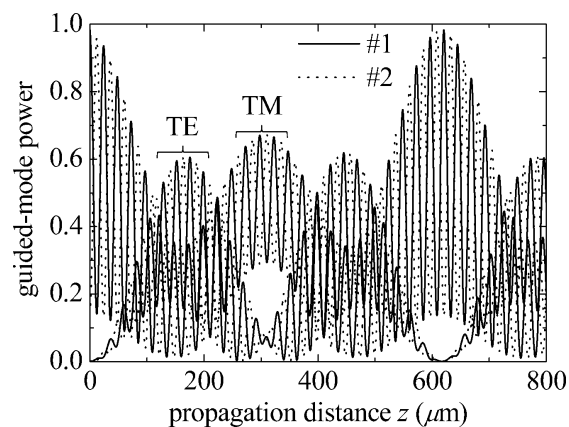

Fig. 5. Guided-mode power against propagation distance for $h=0.4 \mu \mathrm{m}$.

It is seen that the polarization conversion is incomplete. Note that the polarization conversion is caused due to the interference between not only the even supermodes but also the odd supermodes. For example, if the polarization conversion occurs due to the interference between only the even supermodes, the propagating field should return to the input field at $z \simeq 310 \mu \mathrm{m}$ because of the polarization conversion length of $L_{\mathrm{p}(\text { even })}=157 \mu \mathrm{m}$. However, the propagating field does not restore the input field at $z \simeq 310 \mu \mathrm{m}$, because the polarization conversion also occurs due to the interference between the odd supermodes. The former (latter) conversion length observed in the propagating beam analysis is in good agreement with $L_{\mathrm{p}(\text { even })}\left(L_{\mathrm{p} \text { (odd) }}\right)$ calculated by the eigenmode analysis. It follows that efficient polarization conversion cannot be obtained, and the so-called polarization crosstalk is observed. It is also seen that the propagating field almost restores the input field at $z \simeq 620 \mu \mathrm{m}$, because of $4 L_{\mathrm{p}(\text { even })}=2 L_{\mathrm{p} \text { (odd) }} \simeq 620 \mu \mathrm{m}$.

Fig. 5 shows the guided-mode power observed along each waveguide against propagation distance. The polarization crosstalk from the TM mode to the TE mode and vice versa are seen as the field propagates, while transferring the power between the two waveguides.

The field distributions for the TM and TE modes at $y=$ $\pm 0.2 \mu \mathrm{m}$ are illustrated in Figs. 6 and 7, respectively. It is observed that the propagating field couples between Waveguides $\# 1$ and \#2. It is also observed that part of the input TM mode is converted into the TE mode.

\section{B. Polarization Converter Using the Polarization Crosstalk}

In order to make use of the polarization crosstalk constructively, we next determine the vertical spacing $h$ between the

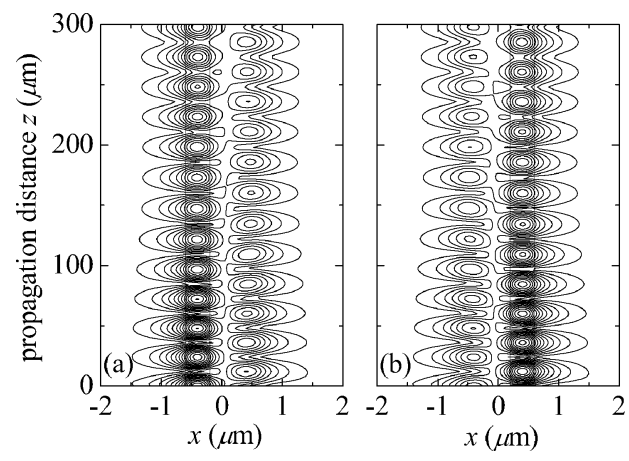

Fig. 6. Field distributions (TM mode). (a) $y=-0.2 \mu \mathrm{m}$. (b) $y=0.2 \mu \mathrm{m}$.

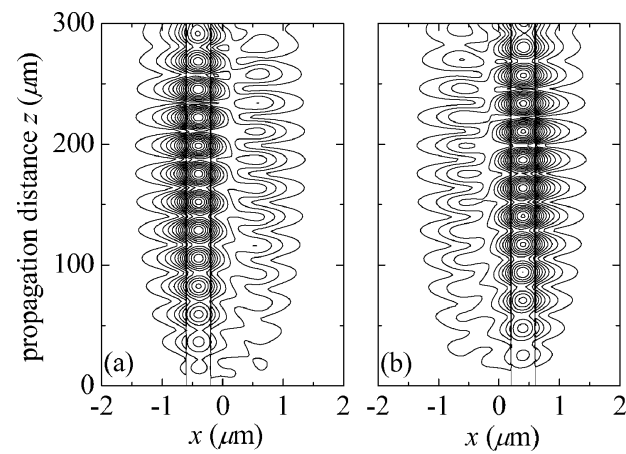

Fig. 7. Field distributions (TE mode). (a) $y=-0.2 \mu \mathrm{m}$. (b) $y=0.2 \mu \mathrm{m}$.

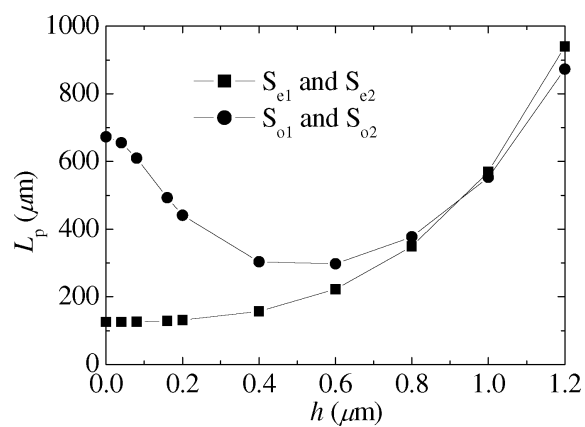

Fig. 8. Polarization conversion length $L_{\mathrm{p}}$ against $h$.

two cores. The complete polarization conversion depends on whether $L_{\mathrm{p}(\text { even) }}\left(L_{\mathrm{p} \text { (odd) })}\right)$ is an odd integer multiple of $L_{\mathrm{p} \text { (odd) }}$ $\left(L_{\mathrm{p}(e v e n)}\right)$ or not. Hence, we first assess the polarization conversion length of the even (odd) supermodes. Fig. 8 shows the polarization conversion length $L_{\mathrm{p}}$ against $h$. It is seen that $L_{\mathrm{p}(\mathrm{even})}$ is very close to $L_{\mathrm{p} \text { (odd) }}$ over a range of $h=0.8 \mu \mathrm{m}$ to $h=$ $1.2 \mu \mathrm{m}$.

Since the polarization conversion occurs due to the interference between the two even (odd) supermodes, the two even (odd) supermodes have to be equally excited for efficient polarization conversion. Therefore, we next assess the overlap integral between each even (odd) supermode and the fundamental TM mode of the isolated waveguide. Fig. 9 shows the overlap integral against $h$. It is seen that, for $h \geq 0.4 \mu \mathrm{m}$, the four supermodes are almost equally excited and the interference between the two even (odd) supermodes occurs with polarization conversion. As a result, we choose a vertical spacing of $h=0.8 \mu \mathrm{m}$, 


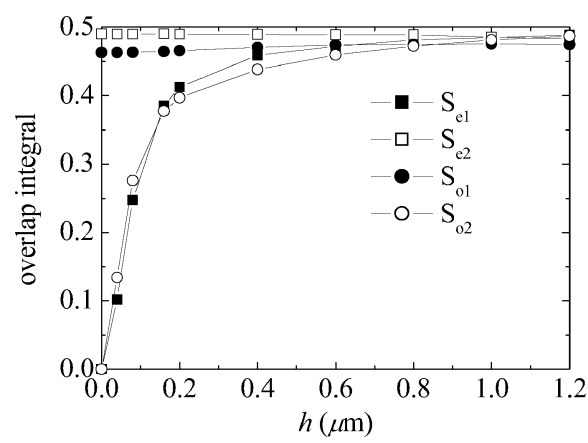

Fig. 9. Overlap integral against $h$.

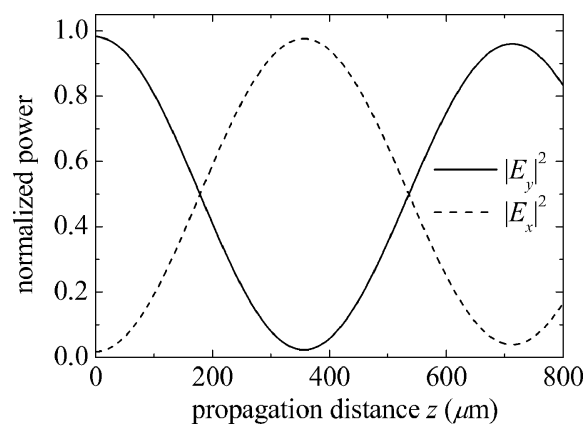

Fig. 10. Normalized power against propagation distance for $h=0.8 \mu \mathrm{m}$.

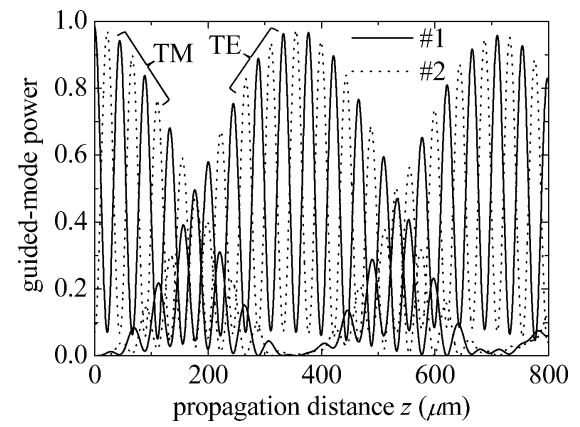

Fig. 11. Guided-mode power against propagation distance for $h=0.8 \mu \mathrm{m}$.

which corresponds to the case where the two cores are stacked diagonally.

Since the vertical spacing $h$ has been determined, we next assess the performance of the converter. Fig. 10 shows the normalized power of each field component for $h=0.8 \mu \mathrm{m}$ as a function of propagation distance. It is seen that the polarization conversion becomes almost complete at $z=360 \mu \mathrm{m}$. This result is again in good agreement with the eigenmode analysis shown in Fig. 8.

Fig. 11 shows the guided-mode power for $h=0.8 \mu \mathrm{m}$ against propagation distance. As expected, almost complete conversion can be obtained at $z=360 \mu \mathrm{m}$, although there still exists the rapid variation of the guided-mode power along the waveguides.

\section{Coupler Composed of Two Rectangular Cores}

So far, it has been found that the almost complete polarization conversion occurs due to the interference between the two

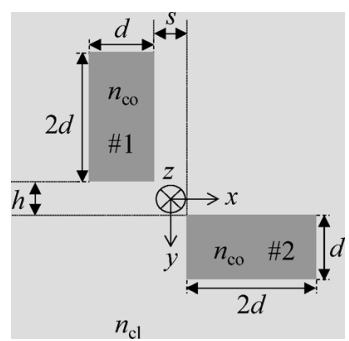

Fig. 12. Configuration of a coupler composed of two rectangular cores.

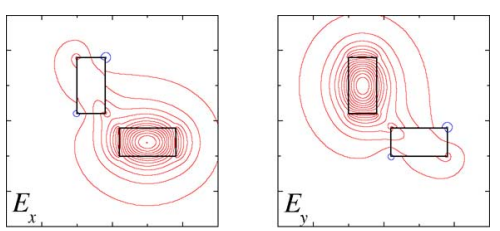

(a)
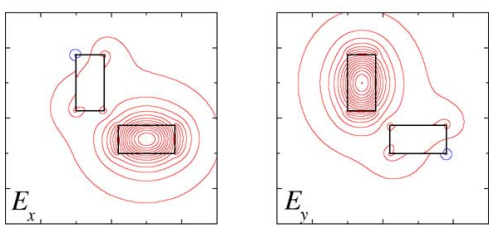

(b)

Fig. 13. Field distributions of the even supermodes. (a) $\mathrm{S}_{\mathrm{e} 1}$ mode. (b) $\mathrm{S}_{\mathrm{e} 2}$ mode.

even (odd) supermodes. We should recall, however, that the interference also occurs between the even and odd supermodes, resulting in the rapid variation of the guided-mode power along the waveguides. To suppress the field variation, we next consider the coupler composed of two rectangular cores shown in Fig. 12. The core with $d=0.4 \mu \mathrm{m}$ is used, and $s$ and $h$ are fixed to be $0.2 \mu \mathrm{m}$. Other configuration and computational parameters are the same as those used in Fig. 1. The vertical spacing between the cores is redefined in Fig. 12.

The eigenmode analysis again shows the existence of two even and two odd supermodes, as shown in Figs. 13 and 14, where the change of the sign of the field is observed. To assess the coupling characteristics, we evaluate the overlap integral between each even (odd) supermode and the fundamental TM mode of Waveguide \#1. Calculation shows that the overlap integral between the even supermode $\left(\mathrm{S}_{\mathrm{e} 1}\right.$ or $\left.\mathrm{S}_{\mathrm{e} 2}\right)$ and the fundamental TM mode is more than $98 \%$, while that between the odd supermode $\left(\mathrm{S}_{\mathrm{o} 1}\right.$ or $\left.\mathrm{S}_{\mathrm{o} 2}\right)$ and the fundamental TM mode is less than $1 \%$. This is due to the fact that the field is predominantly distributed in one of the two waveguides. For example, the TM-mode field is distributed in Waveguide \#1 for the two even supermodes, and the TM-mode field is distributed in Waveguide \#2 for the two odd supermodes. This means that, when Waveguide \#1 is excited with the fundamental TM mode, only the two even supermodes are excited. As a result, the polarization of the input field is converted, as the field propagates, while no significant interference occurs between the even and odd supermodes. 

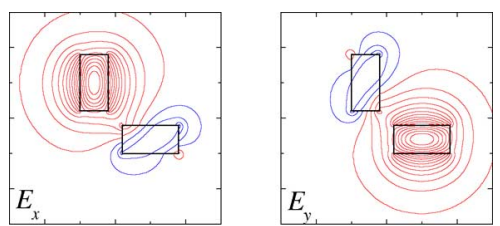

(a)
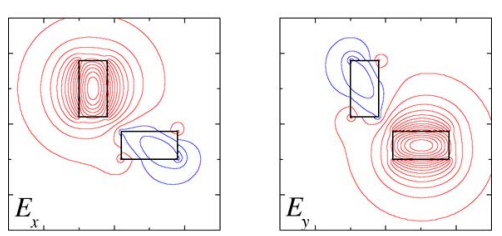

(b)

Fig. 14. Field distributions of the odd supermodes. (a) $\mathrm{S}_{\mathrm{o} 1}$ mode. (b) $\mathrm{S}_{\mathrm{o} 2}$ mode.

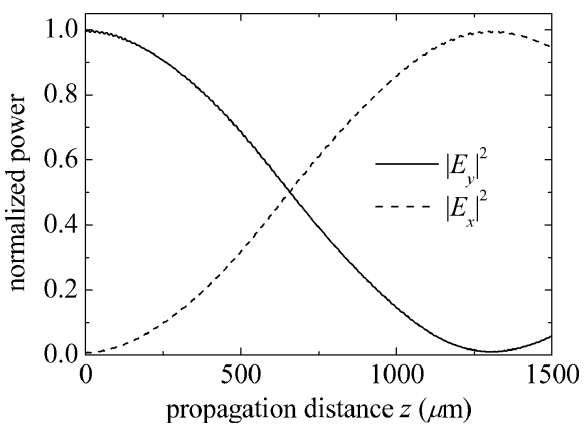

Fig. 15. Normalized power against propagation distance.

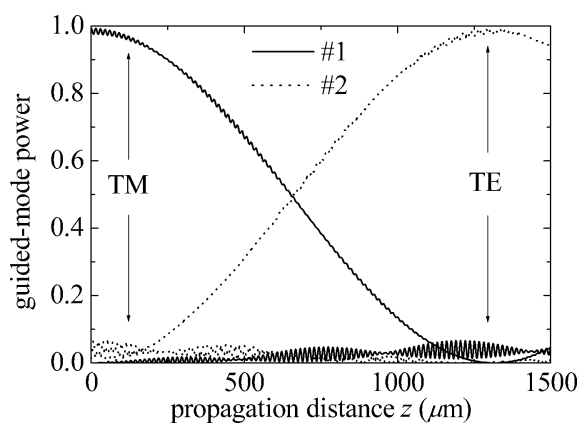

Fig. 16. Guided-mode power against propagation distance.

Fig. 15 shows the normalized power of each field component as a function of propagation distance. It is seen that almost complete conversion can be obtained at $z=1300 \mu \mathrm{m}$.

The effect of suppressing the rapid variation of the power is clearly seen in Fig. 16, which shows the guided-mode power as a function of propagation distance. As expected, almost complete mode conversion can be obtained at $z=1300 \mu \mathrm{m}$, while suppressing the rapid power transfer between the waveguides.

\section{CRossing-WaVeguide-Type Polarization CONVERTER}

The study in the previous sections has showed that only the excitation of either the even supermodes or the odd supermodes is necessary to realize the polarization converter. It is readily expected that the spacing $s$ should be small to achieve a short conversion length. However, this results in the excitation of undesirable supermodes with the rapid power variation along the

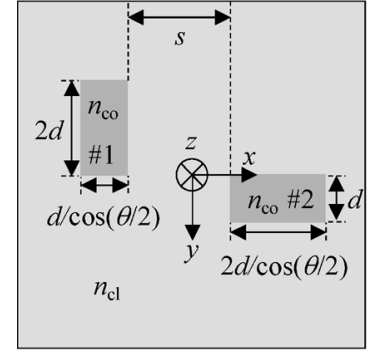

(a)

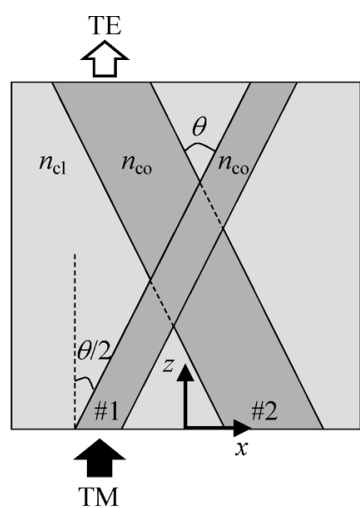

(b)
Fig. 17. Configuration of a crossing-waveguide-type polarization converter. (a) Cross section. (b) Top view.

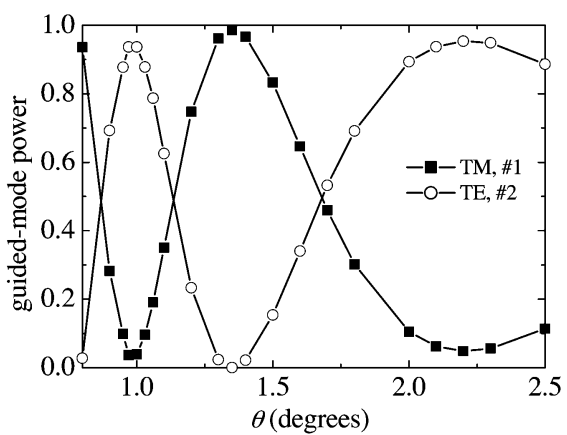

Fig. 18. Guided-mode power against crossing angle.

waveguides. In this section, we, therefore, develop a polarization converter composed of crossing waveguides. Fig. 17 shows the configuration of a crossing-waveguide-type polarization converter. We excite the field of the TM mode from Waveguide $\# 1$ and extract the TE mode in Waveguide \#2. The crossing angle is designated as $\theta$. The configuration parameters are taken to be the same as those of Fig. 12. The vertical spacing between the cores is taken to be $h=0 \mu \mathrm{m}$. The sampling widths are set to be $\Delta x \simeq \Delta z=0.01 \mu \mathrm{m}$ and $\Delta y=0.025 \mu \mathrm{m}$.

We should again recall that when the square cores are used, the interference occurs between the even and odd supermodes, with subsequent rapid variation of the guided-mode power along the waveguides. It follows that the power with polarization conversion is, in general, distributed in both waveguides. The waveguide to which the power is outputted also depends on the crossing angle. This results in a complicated design procedure. Therefore, the suppression of the rapid variation of the power using the rectangular cores is important in practice.

To assess the performance of the polarization converter, we evaluate the guided-mode power. Fig. 18 shows the guidedmode power as a function of crossing angle $\theta$. We calculate the guided-mode power using the propagating field at the output and the fundamental TM (TE) mode of Waveguide \#1 (\#2). It is found that the minimum guided-mode power of the TM mode is calculated to be 0.04 at $\theta=1.0^{\circ}$ and 0.05 at $\theta=$ $2.2^{\circ}$, respectively. In contrast, it is also found that the maximum guided-mode power of the TE mode is calculated to be 0.94 at $\theta=1.0^{\circ}$ and 0.95 at $\theta=2.2^{\circ}$, respectively. For $\theta=2.2^{\circ}$, the device length is shorter than that for $\theta=1.0^{\circ}$. We, therefore, choose $\theta=2.2^{\circ}$. 


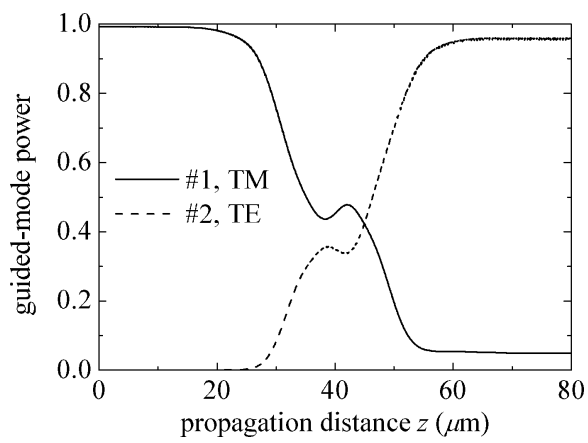

Fig. 19. Guided-mode power against propagation distance.

Fig. 19 shows the guided-mode power for $\theta=2.2^{\circ}$ as a function of propagation distance. It is observed that the incident TM mode is converted into the TE mode at $z \simeq 80 \mu \mathrm{m}$ (the polarization conversion mainly occurs over a region of 20-60 $\mu \mathrm{m}$ ). It should be noted that the device length of this converter is significantly shorter than that of the coupler composed of the two rectangular cores shown in Fig. 12.

\section{CONCLUSION}

We have evaluated the polarization coupling between strongly guiding waveguides stacked laterally using the FV-BPM. The eigenmode analysis shows the existence of four supermodes, suggesting the polarization conversion due to the interference between the two even (odd) supermodes. We evaluated the polarization conversion length of the even (odd) supermodes. For the propagating beam analysis, it is confirmed that the polarization conversion occurs due to the interference between not only the even supermodes but also the odd supermodes. The conversion lengths are in good agreement with those calculated by the eigenmode analysis. In order to make use of the polarization crosstalk constructively, we next determine the vertical spacing between the two waveguides. For the propagating beam analysis, the input TM-mode field is converted into the TE-mode field with a guided-mode power of $98 \%$, when the two waveguides are stacked diagonally. We have also found that a coupler composed of two rectangular cores becomes an efficient polarization converter. In the case of the rectangular cores, the polarization of the input field is converted, as the field propagates, while no significant interference occurs between the even and odd supermodes. Finally, a crossing-type polarization converter has been analyzed. The polarization converter, which consists of the crossing rectangular waveguides, leads to a reduction in the device length.

\section{REFERENCES}

[1] S. T. Chu, B. E. Little, W. Pan, T. Kaneko, S. Sato, and Y. Kokubun, "An eight-channel add-drop filter using vertically coupled microring resonators over a cross grid," IEEE Photon. Technol. Lett., vol. 11, no. 6, pp. 691-693, Jun. 1999.

[2] B. E. Little and S. T. Chu, "Theory of polarization and conversion in vertically coupled microresonators," IEEE Photon. Technol. Lett., vol. 12, no. 4, pp. 401-403, Apr. 2000.

[3] B. Liu, A. Shakouri, P. Abraham, and J. E. Bowers, "Optical add/ drop multiplexers based on X-crossing vertical coupler filters," IEEE Photon. Technol. Lett., vol. 12, no. 4, pp. 410-412, Apr. 2000.

[4] C. Fietz and G. Shvets, "Nonlinear polarization conversion using microring resonators," Opt. Lett., vol. 32, no. 12, pp. 1683-1685, 2007.

[5] J. Yamauchi, G. Takahashi, and H. Nakano, "Full-vectorial beam-propagation-method based on the McKee-Mitchell scheme with improved finite-difference formulas," J. Lightw. Technol., vol. 16, no. 12, pp. 2458-2464, 1998
[6] Y. L. Hsueh, M. C. Yang, and H. C. Chang, "Three-dimensional noniterative full-vectorial beam propagation method based on the alternating direction implicit method," J. Lightw. Technol., vol. 17, no. 11, pp. 2389-2397, 1999.

[7] J. Yamauchi, N. Shibuya, and H. Nakano, "Coupling characteristics between strongly guiding waveguides stacked laterally," in Proc. Integ. Photon. Nanophoton. Res. Appl., Salt Lake City, UT, Jul. 2007, paper IWB6.

[8] T. Yamazaki, J. Yamauchi, and H. Nakano, "Eigenmode analysis of a light-guiding metal line loaded on a dielectric substrate using the full-vectorial imaginary-distance beam-propagation method," Trans. IEICE, vol. J90-C, no. 6, pp. 465-473, Jun. 2007.

[9] D. Yevick and W. Bardyszewski, "Correspondence of variational finitedifference (relaxation) and imaginary-distance propagation methods for modal analysis," Opt. Lett., vol. 17, no. 5, pp. 329-330, 1992.

[10] M. R. Watts and H. A. Haus, "Integrated mode-evolution-based polarization rotators," Opt. Lett., vol. 30, no. 2, pp. 138-140, 2005.

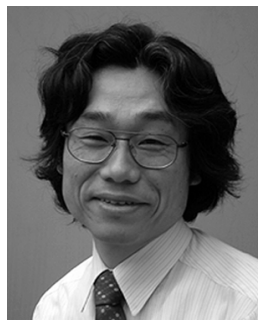

Junji Yamauchi (M'84-SM'08) was born in Nagoya, Japan, on August 23, 1953. He received the B.E., M.E., and Dr.E. degrees from Hosei University, Tokyo, Japan, in 1976, 1978, and 1982, respectively. From 1984 to 1988, he served as a Lecturer in the Electrical Engineering Department of Tokyo Metropolitan Technical College. Since 1988, he has been a member of the faculty of Hosei University, where he is now a Professor. His research interests include optical waveguides and circularly polarized antennas. He is the author of Propagating Beam Analysis of Optical Waveguides (Baldock, Hertfordshire, U.K.: Research Studies Press, 2003).

Dr. Yamauchi is a member of the Optical Society of America and the Institute of Electronics, Information and Communication Engineers of Japan.

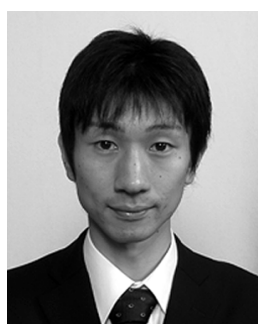

Noriyuki Shibuya was born in Chiba, Japan, on June 24, 1983. He received the B.E. and M.E. degrees from Hosei University, Tokyo, Japan, in 2006 and 2008, respectively.

In 2008, he joined Sharp Corporation, Kameyama, Japan.

Mr. Shibuya is a member of the Institute of Electronics, Information, and Communication Engineers (IEICE) of Japan.

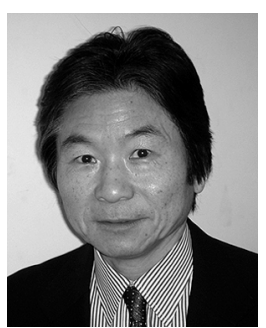

Hisamatsu Nakano (M'75-SM'87-F'92) was born in Ibaraki, Japan, on April 13, 1945. He received the B.E., M.E., and Dr.E. degrees in electrical engineering from Hosei University, Tokyo, Japan, in 1968, 1970, and 1974, respectively.

Since 1973, he has been a member of the faculty of Hosei University, where he is now a Professor of electronic informatics. His research topics include numerical methods for low- and high-frequency antennas and optical waveguides. He has published more than 200 refereed journal papers, more than 200 international symposium papers, and more than 750 national symposium papers. He is the author of a book entitled Helical and Spiral Antennas (New York: Research Studies Press, Wiley, 1987) and the coauthor of Analysis Methods of Electromagnetic Wave Problems, Volume Two (Norwood, MA: Artech House, 1986). In addition, he is the author of Helical and Spiral Antennas, Encyclopedia of Communications (New York: Wiley, 2002).

Dr. Nakano received the IEE International Conference on Antennas and Propagation Best Paper Award and the IEEE Transactions on Antennas and Propagation Best Application Paper Award (H. A. Wheeler Award), in 1989 and 1994, respectively. In 1992, he was elected as an IEEE Fellow for contributions to the design of spiral and helical antennas. In 2001, he received the Award of Distinguished Technical Communication (from the Society for Technical Communication, USA) and the Science and Technology Progress Award (from Hangzhou, China). He is an Associate Editor of several journals and magazines, such as Electromagnetics, IEEE ANTENNAS AND PROPAGATION Magazine, IEEE ANTENNAS AND Wireless Propagation LetTers, and Asian Information-Science-Life. 